\section{Towards the digital library: how relevant is e-lib to practitioners?}

\author{
Andrew Green, (National \\ Library of Wales)
}

One day last month I had a glimpse of the future.

I was in my office with some library colleagues and two members of the Department of Physics. We were there to discuss electronic journals, but the conversation broadened to include all scholarly communication in the area of particle physics research. After a few minutes an awful truth dawned: that in the eyes of the physicists I and for that matter all of my colleagues - were utterly redundant to their information needs. Those needs were supplied almost entirely by Paul Ginsparg's electronic physics archive at Los Alamos, now mirrored in this country at the University of Southampton (1). It was to this online archive that they turned for existing literature, and it was to the same archive that they posted their own research. Not only were all other libraries redundant, so too, almost, were publishers: editing and dissemination of articles were done by the researchers and the Archive, and only refereeing remained to them of their traditional roles.

The physicists rather overegged their cake by claiming that all other academic disciplines would follow their example within 18 months, but they had succeeded in making a group of academic librarians aware, in the most arresting way, of the complete surrender of print to digital information.

Such moments of revelation are rare. More usually the impact on librarians of new technologies, and technological programmes like the Electronic Libraries Programme (e-lib), is more varied, more diffuse, and more difficult to assess.

E-lib, of course, is far from finished, and any judgement now of its relevance to libraries and their staffs can only be provisional. Even when all of the projects have come to the end of their lives - and hopefully many will continue as services long after the initial funding runs out - it will be a long time before final assessments can be made.

Another difficulty is that e-lib is so large a programme, with over 60 separate projects, that one person cannot, without a great deal of effort, grasp it in all its variety. Each project could have a different profile of influence and impact, and on different sets of practitioners - those working in libraries which were primary or secondary sites of the projects, and those in libraries not directly connected. Library staffs, of course, are by no means the only constituency addressed by the Programme, and it would be unfair to judge its performance on its impact on them alone.

All I can do today is to give you my own views about how relevant e-lib has been so far to life as it is lived in our own library - along with the views of some of my colleagues. I recently asked all staff in Library \& Information Services in Swansea two simple questions:

'Do you think the impact of e-lib on your work has been (a) substantial, (b) moderate or (c) negligible?' and, 'If you answered (a) or (b), where do you think the impact was felt?'

The responses were revealing, and I shall try to weave quotations from them into what follows.

I'd now like to consider the nature, aims and methodology of e-lib from a practising librarian's point of view; then glance at the work of the main programme areas; and finally offer you some thoughts on cultural influence and cultural change.

Beginning at the beginning, and being a literalminded person, I thought it would be helpful to remind myself of the original aims of the Programme. Much to my surprise - and it never occurred to me to be surprised at the time, three years ago - I found that there were no publicly stated aims. The two key JISC documents 
announcing the establishment of e-lib, Circular 2/94 and Circular 4/94, conspicuously fail to say what the effect the spending of $£ 15$ million was intended to be. It was clear what the foci of the Programme were to be - they were taken almost wholesale from the IT chapter of the Follett Report (2). It was clear from the second circular what the functions of FIGIT, the Programme's controlling committee, were to be. But there was nothing to say what objectives the Programme itself was to have, where its influence was to be felt, and how its results were to be diffused through the sector. In retrospect it is greatly to the credit of the members of the FIGIT and the e-lib Directorate that coherent answers have been sought and mostly given to these questions since 1994.

As it happened it was the methodology adopted by FIGIT that in large part determined how the Programme would approach its aims. The policy of, to quote Mao Tse-Tung, 'letting a hundred flowers blossom and a hundred schools of thought contend' - setting up numerous parallel projects in a single programme area - together with the preference for consortia, has meant that over sixty academic libraries have experienced at first hand at least one aspect of the developing electronic library. This is in strong contrast to the US Digital Libraries Programme, with its small number of large projects. If one includes libraries which were not direct recipients of e-lib money, but associated partners, it is likely that very few UK academic libraries have not been touched by at least one e-lib project.

The building-in of redundancy had another advantage beyond a guaranteed immediate impact on libraries. It was certain that not all projects would succeed. In Darwinian terms only the fittest would survive. But the 'hundred flowers' approach also had its downside. Each project was smaller and had fewer resources:

I still have a general feeling that there are TOO MANY e-lib projects around, and that there must have been quite a lot of duplication of effort (especially in the electronic short loan area). I wonder if it was all spread a bit too thinly?

[Senior manager]
Many teams found themselves tackling, in parallel and with inadequate resources, the same or very similar problems, technical and non-technical. One suspects that problems like copyright, authentication and standards might have been best tackled by the more concentrated application of elib funds at the beginning of the Programme, rather than left for individual action projects to encounter in isolation.

The other striking feature of the projects from the beginning was that they were intended to be, by the end of their lives, 'whole packages'; that is, each was supposed to result in a working product or service, of practical benefit to the library and information communities.

Like the 'hundred flowers' approach the effect of the 'whole package' policy, if successful, would have an immediate impact on library practitioners. The product of the projects would be not prototypes or demonstrators, but real, immediately usable services.

Finally, a third prominent feature of e-lib, at least in recent times, has been the very strong emphasis on evaluation, both formative and summative, and on dissemination, in the double sense of devoting a whole programme area, Training \& Awareness, to disseminating the good news about the digital library, and of insisting that each project gives sufficient attention to telling the world about its existence, its successes and its difficulties.

All three features of e-lib, it seems to me, would tend to improve its chances of making a real impact on libraries and librarians, even at this early stage.

It's now time to turn briefly to the programme areas and projects themselves, and try to estimate the extent to which working librarians see them as relevant to their work. Here I rely heavily on the contributions of my colleagues in Swansea, whom I mentioned earlier. For the sake of speed I'm going to group the programme areas together into 'clumps'.

First, the area of electronic journals, pre-prints and digitisation - essentially, those projects aimed 
at helping researchers. These are small in number and experimental in nature, and for those reasons can hardly be said to have made any significant impact yet on libraries:

I look at the ejournals and related areas such as digitisation from time to time, but nothing much seems to happen.

[Senior manager]

They have also been overshadowed by the much larger and bolder Periodicals Pilot Site Licence Initiative (PSLI), established in 1995 by the Higher Education Funding Councils. Although this too has yet to reach maturity, by virtue of its size (over $£ 2 m$ ), the universality of its scope (all $\mathrm{HE}$ institutions) and the eager participation of publishers within it, it is more likely in my view to have a decisive effect on professional and scholarly practice than the e-lib projects.

The PSLI journals, of course, are all parallel versions of existing print titles, and assume a continuing intermediary role for the commercial periodical publisher. As my physics example showed, a quite different model, dispensing with the publisher, is entirely possible, at least in small, well-organised disciplines, and the e-lib projects may well have a part to play in developing this alternative model.

In this area it seems that the e-lib's significance lies less in immediate practical influences than on pointing to the potential of new ways of organising scholarly communication. The work of the existing projects would need to be scaled up considerably before their benefits could be felt in institutions.

In the area of digitisation, although increased awareness is already obvious

e-lib projects play a significant part in a growing awareness of the possibilities provided by digitisation, etc.

[Subject librarian]

The small number of e-lib projects may be overshadowed by larger-scale service initiatives. I'm thinking here of JISC's new Digitisation Centre and of the JSTOR service, which originated in the US and is likely to become available through JISC in the UK soon.

Secondly, electronic short-loan and on-demand publishing projects. By their nature these have had few effects in non-participating libraries. On the other hand many eyes in libraries throughout the country must be watching their progress closely:

My hope is that e-lib will finally crack the general provision of electronic short loan. Everyone (apart from the publishers) feels that this is a Good Thing and technically feasible. It needs something like a variety of e-lib projects ... to give the final push towards this.

[Senior manager]

Success in this area would solve one of the most intractable problems faced by academic libraries: how to provide all students on a course with copies of required readings simultaneously and when they are needed. Our continued failure to achieve this is arguably the cause of more student discontent than any other library issue. Again, though, a larger-scale programme is needed to translate the work of the existing projects into working systems where electronic delivery to students starts to replace print.

Third, access to network resources, described by the Tavistock Institute as 'the most stable and coherent element of the [Electronic Libraries] Programme' (3). One can already describe this as one of e-lib's success stories. The subject gateways have embedded themselves into professional practice very swiftly:

[I] use OMNI as a way of updating on the progress and developments of new internet resources relevant to students and staff at the department. OMNI is ncluded in every session that I've run with staff and students ... the address of the OMNI gateway is included on all of the guides produced for students.

[Subject librarian]

... the EEVL gateway for engineers springs to mind as being useful.

[Subject librarian] 
I have shown researchers in history IHR-Info which has many links to useful resources for historians

[Subject librarian]

... the establishment of information gateways ... thus prov[ides] librarians with high quality tailor-made resources which we can promote to staff and students.

[Subject team member]

Subject gateways and general proselytising on a subject basis like SOSIG and EEVL I suspect have had a major impact on their academic communities

[Senior manager]

What is particularly valued, it seems, is the labour-intensive quality control the services impose on the sources they describe.

One can even claim that the subject gateways have saved libraries money:

I first heard about the National Library of Medicine's initiative of allowing free access to Medline via OMNI. (We were looking at ways of allowing all of our [nursing library] sites to access Medline, which would cost around $£ 5,000$.) OMNI's website had a review of this version of Medline (PubMed) - this helped us in the decision to use the NLM's PubMed (and saving $£ 5,000$ !).

[Subject librarian]

These services, and their parents, BUBL's LINK and NISS's Directory of Networked Resources, by doing the task on behalf of the whole community, and much more efficiently than could any individual library, have relieved many libraries of the burden of growing their own 'subject trees'. (Incidentally is it not high time that the BUBL and NISS services were combined into a single and better service? The continuing rivalry between the two, both funded by the same agency, JISC, is incomprehensible to most working librarians.)

My fourth group is electronic document delivery. Here the acid test of the projects will whether any of them can in time supplant the British Library Document Supply Centre as the dominant supplier to most academic libraries of material not held locally. The omens are not good. Only one of the projects, LAMDA, has yet reached the stage of a working service, and there must be some reason to doubt whether the full economic costs of document supply are being calculated and reflected in charges to users.

It is tempting to take an 'old Labour' view, one at odds with the prevailing faith in the competitive market, that we should be making the most of what other countries envy us for, a highly efficient and successful national document supply service. Instead of seeking to undermine it by pumppriming fledgling competitors, perhaps we should be putting higher education money and energy into improving and extending the DSC service and increasing our stake in its success and operation.

Finally, the training and awareness projects. Since most of these were expressly aimed at influencing librarians (and others) in the field, one might expect them to be mentioned more often than others, and indeed they were in my small sample:

The EduLib project has set standards for library training even if the initial idea of pyramid training was flawed. I was impressed with their taster workshops and intend to use some ideas in teaching. NetLinks has also had practical benefits.

[Subject Team member]

For me EduLib especially was useful for personal extra training ... [Subject Team member]

I have attended three workshops provided under the EduLib programme ... The information and techniques learned at these events will have an impact when I start to plan and carry out any teaching. I think that the most valuable aspect of these courses was in the provision of an underlying structure within which one could work. This gives one the confidence that you have at least covered all the essential procedures.

[IT manager]

After a slightly ropey start, the training \& awareness programmes like Netskills and EduLib now seem to be delivering the goods. I suspect that the methodology doesn't matter as 
Towards the digital library: Andrew Green much as the heightened awareness.

[Senior manager]

My own feeling about the training and awareness projects is that, as in some other areas, there are too many of them: a more concentrated effort with more resources could, one feels, have had a more profound effect on the library community.

Netskills seminars, for example, are rarely held anywhere remotely accessible to staff in Swansea. The NetLinks project has been torn between having a general, shallow effect and concentrating its efforts on a few libraries.

So much for the individual programme areas. What has been the general impact of e-lib on my LIS colleagues? Of the fourteen responses just two were from IT staff; one assessed the impact as negligible, the other as moderate. The manager of our Recording Centre for the Blind could detect no impact, but wondered about the potential of the Programme to benefit visually impaired people. Among the librarians the scores were: substantial impact: 2, moderate impact : 6, negligible impact: 3 .

One factor in the lack of visibility of e-lib projects among practitioners I've already mentioned: the 'hundred flowers' policy. With so many plants on the forest floor, all competing for scarce sunlight, it's not surprising that only a few larger and hardier or flashier varieties really thrive and are seen. This problem will become more acute as the projects reach the end of their lives and all seek simultaneously to disseminate the results of their work to the rest of us.

The latest phase of e-lib, so-called e-lib 3, implicitly acknowledges the lack of articulation in the programme to date in its desire to see 'hybrid library' projects that bring together the scattered elements of the programme areas.

And what will happen when the money does run out? 'Exit strategies', which all projects are being encouraged to consider, is a term that conjures up assisted suicide rather than prolonged life. Will the useful services survive? Even more important, how will the useful projects spawn similar services, equally needed? I'd like now to consider the difficult question of the cultural influence of the Programme. By cultural influence I mean specifically the ability to affect the behaviour and outlook of individuals and organisations in such a way that new ways of working become embedded and future change becomes autogenic, that is, without the need for further external stimulus.

It is extremely difficult to assess the cultural influence of e-lib on libraries, for three reasons: first, libraries had been moving in electronic directions, admittedly at different speeds, well before e-lib started to take effect, witness the rapid spread of CD-ROMs and BIDS, and the rise of converged services combining libraries and computer centres. These innovations developed their own momentum. Second, there are many other external stimuli working in parallel in the same direction. To take some examples from national initiatives, the Periodicals Pilot Site Licence Initiative, CHEST dataset deals and the Non-Formula Funding humanities research collections initiative of the Funding Councils have all left their mark on library practice; arguably a more profound mark than e-lib. (It is sometimes tempting to wonder whether spending e-lib's $£ 15$ million on these and other substantial initiatives, like retrospective catalogue conversion, would have resulted in a greater and more practical achievement.) Thirdly, influence may be indirect rather than direct, and those who feel it may not be aware that the source was in fact some aspect of e-lib.

There is a further difficulty, to do with the ability of librarians to effect cultural change in their immediate and wider environments. Even if staff are well-positioned and well-disposed to change, the climate they work in may militate against that change being internalised. I'm thinking, for example, of the current financial crisis in higher education, that allows little room for the kind of experiments and initiatives that are needed to put e-lib's insights and advances into practice locally. Similarly, severe problems await librarians working in institutions resistant or indifferent to the claims of the electronic library: the commitment of senior managements, whether or not expressed in formal strategies or policies, is crucial to making rapid progress. 
This type of thing [seminars and other publicity on e-lib projects] has probably contributed to changing the culture in academic libraries to some extent, but has highlighted the problems of getting support at institutional level.

[Senior manager]

Another, paradoxical example is convergence. Though convergence should result in greater opportunities for applying IT to library services, the process of converging library and computer organisations, I suspect, can result in a diversion of energies away from innovation in service into internal structural reorganisation.

I seem to be arguing myself into a corner - into admitting that there can be no way of judging whether e-lib is seen as relevant to professional practice. Perhaps projects like IMPEL2 (4) and other supporting studies (5) will be able to shed more light on the problem. But let me now venture some personal judgements - some supported by evidence from my colleagues about the influence of e-lib.

1 First, the level of awareness of e-lib and its importance is without doubt widespread among working librarians.

[e-lib has] generated a great deal of discussion every conference/meeting has at least one e-lib project mentioned

[Subject Team member]

Some of the training and awareness projects, notably EduLib, are succeeding not only in diffusing knowledge of the technologies but also in suggesting to librarians how to spread that knowledge wide within their institutions. Much still needs, to be done, however, to draw the attention of working librarians to the essential results of most projects.

2 The existence of e-lib has allowed librarians to focus the attention of their institutions on libraries and their potentialities. It has been a convenient and familiar peg on which chief librarians can hang arguments addressed to their vicechancellors and directors of finance about the modernity of libraries and their continuing relevance to learning and research.

3 e-lib has the potential to solve some of the outstanding problems that have plagued academic librarians for many years: for example, how to supply students with electronic copies of basic recommended readings. But in many cases larger-scale work is needed before real cultural change is guaranteed.

Through the establishment of subject gateways elib is succeeding in applying traditional librarianly skills to a new sphere of knowledge, the World Wide Web. It is very important that ways are found of prolonging the current projects when current funding ceases, and of engendering other gateways.

In other areas, like electronic journals, though valuable experience may be gained, the pace is likely to be set by external commercial forces.

4 In some respects e-lib has failed to make the right connections. It has failed to make any real connection with other IT-related national programmes in higher education, especially the Computers in Teaching Initiative (CTI) and Teaching and Learning Technology Programme (TLTP) - despite the fact that these too are concerned to spread lessons from exemplars around the country and affect the prevailing culture. This failure to locate itself in the universe of HE IT might be one reason why it features so little in the thinking of the Dearing Report.

Another failed connection, if the Swansea experience is typical, is with our fellow professionals in computer service departments, where awareness of e-lib seems to be low. This matters because, especially in converged services, well-informed co-operation between librarians and computer staff could yield early results.

5 The shape of the e-lib programme is based on functions - short loan, or network navigation. Few projects therefore took as their starting point particular groups of library user and followed a more holistic line. For example, distance learners, archive users or visually impaired users could perhaps have had programme areas devoted to 
finding electronic solutions to their various needs and problems.

E-lib's shape was also determined, at least at the outset, by the recommendations of the Follett Report. Some may feel that Follett was followed too rigidly. For example, was it wise to reject all thought of funding projects based on library automation systems, on the grounds that the commercial sector would fund all necessary innovation? Chances may have been lost to stimulate the creation of distributed virtual libraries, and integrated bibliographical systems including library and bookshop data.

6 The expertise gained by staff working on e-lib projects is a certain gain for the sector. A small army of mainly young librarians and information professionals will have gathered invaluable experience it would be good to think that they will not be lost to the profession but will find employment in libraries and spread their expertise far and wide.

Many libraries will have built up experience they did not possess before of the processes of constructing and costing project proposals and of project management - experience that will be helpful in an age that increasingly favours handto-mouth above assured funding:

... there must be lots of people in jobs (albeit temporary jobs) which are different from your run-of-the-mill library stuff - project management, business plans, bidding for . funding etc. So the programme must have broadened our experience as a profession ... [Senior manager]

7 Finally, one wholly positive outcome of e-lib has been its creative contribution to professional jargon. Would the world not be poorer without the invention of terms like 'clumps' and the 'hybrid library', and acronyms like SOSIG, JILT, and my own favourite (and the only e-lib project named after a blues by Howlin' Wolf), EEVL?

To sum up, despite some reservations I believe that when the time comes for judgement the Electronic Libraries Programme will prove to have had a powerful catalytic effect on professional library practice. Without it, our knowledge would be weaker, our standing lower, and our confidence to face the future less. Despite what our friends from the Physics Department think, change is most often incremental than cataclysmic, and e-lib will have pushed us a few more steps in the right direction.

\section{ACKNOWLEDGEMENT}

Many thanks to my colleagues in Library \& Information Services in the University of Wales Swansea, who contributed much, directly and indirectly, to the content of this article.

\section{REFERENCES}

1. Physics e-print Archive mirror: http://www.soton.ac.uk/

2. Joint Funding Councils' Libraries Review Group, Report. Bristol: Higher Education Finding Council for England, 1993, Chapter 7.

3. Tavistock Institute, Evaluation of the Electronic Libraries Programme. Policy mapping study: the set-up, operation and content of the Electronic Libraries Programme. August 1996. http://www.ukoln.ac.uk/services/elib/papers/tavistock/policy-mapping/

4. Day, J, Edwards, C and Walton, $G$ The impact on people of electronic libraries: monitoring organizational and cultural change in UK higher education. htep://www.unn.ac.uk/ liy8/impel2/cni4.htm

5. Davies, C, Scammell, A and Hall, M e-lib: changing the lightbulb - er, the culture Ariadne, issue 10, 1997 [electronic version only], http://www.ariadne.ac.uk/issue /0/cultural/ 